\title{
Dual Mechanical and Pharmacological Thromboprophylaxis Significantly Decreases Risk of Pulmonary Embolus after Laparotomy for Gynecologic Malignancies
}

\author{
JMV Nguyen $_{a}$, LT Gien ${ }_{b}$, A Covens $_{b}$, R Kupets , R Osborne $_{b}$, M Sadeghi ${ }_{c}$, AB Nathens N , D Vicus . .
}

Division of Gynecologic Oncology, University of Toronto, Toronto, Canada Division of Gynecologic Oncology, Sunnybrook Health Sciences Centre, University of Toronto, Toronto, Canada Department of Surgery, Sunnybrook Health Sciences Centre, University of Toronto, Toronto, Canada. Division of General Surgery, Department of Surgery, Sunnybrook Health Sciences Centre, University of Toronto, Toronto, Canada.

\section{BACKGROUND}

- Thromboembolic events are the second most common cause of mortality in patients living with cancer.

- Patients undergoing laparotomies for gynecologic malignancies are high risk of venous thromboembolism (VTE) with reported rates as high as $13 \%$. VTE can increase mortality by up to 2 -fold.

- Currently, there is no consensus for perioperative thromboprophylaxis specific to Gynecologic Oncology.

- Dual thromboprophylaxis with mechanical and pharmacological methods is recommended by the American College of Chest Physicians and American Society of Clinical Oncology for high-risk post-operative patients.

\section{OBJECTIVES}

- The primary objective was to compare rates of symptomatic pulmonary embolus (PEs) within 30 days of surgery before and after implementation of dual thromboprophylaxis.

- The secondary objective was to identify risk factors for PEs.

\section{METHODS}

- The Gynecologic Oncology division at Sunnybrook Health Sciences Centre (SHSC) in Toronto, Canada, implemented a three-fold strategy in $12 / 2017$ for patients undergoing laparotomies.

- Prospective cohort study of patients who underwent laparotomies for a suspected or confirmed malignancy by a Gynecologic Oncology surgeon between $12 / 2017$ to $10 / 2018$, with comparison to a historical cohort who underwent surgery between 01/2016 to $11 / 2017$.

\section{Three-fold strategy}

$\checkmark$ SCDs were applied prior to start of surgery and worn during hospital stay

$\checkmark$ LMWH during admission

$\checkmark$ LMWH for an extended duration of 28 days when a malignancy was confirmed.

\section{RESULTS}

534 patients were included: there were 371 and 163 laparotomies pre-and postintervention.

\section{Demographic and operative details}

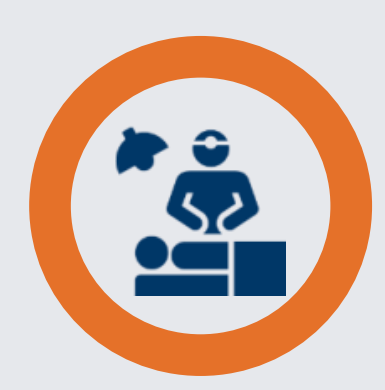

There were more cytoreductive surgeries in the postintervention cohort, but when comparing by surgical complexity score, there were no differences. All other patient and surgical details were similar in both groups.

\begin{tabular}{|lccc|}
\hline \multicolumn{4}{|c|}{ Demographic and Operative Details } \\
\hline & $\begin{array}{c}\text { Pre- } \\
\text { intervention } \\
n=371\end{array}$ & $\begin{array}{c}\text { Post- } \\
\text { intervention } \\
n=163\end{array}$ & p- value \\
Age (median, range) & $62(21-89)$ & $62(24-98)$ & 0.75 \\
BMI (median, range) & $28(17-63)$ & $29(17-60)$ & 0.86 \\
Diabetes & $54(15 \%)$ & $19(12 \%)$ & 0.41 \\
Smoking & $29(8 \%)$ & $19(12 \%)$ & 0.19 \\
Confirmed malignancy & $334(90 \%)$ & $143(88 \%)$ & 0.55 \\
Advanced stage & $236(64 \%)$ & $105(64 \%)$ & 0.92 \\
Cytoreductive surgery & $186(50 \%)$ & $50(28 \%)$ & 0.0001 \\
Surgical complexity score** & $3(1-14)$ & $3(1-13)$ & 0.82 \\
(median, range) & low (low to & low (low to & 0.87 \\
Surgical complexity score & high) & high) & \\
group ** (median) & $350(50-9000)$ & $350(50-7000)$ & 0.92 \\
Blood loss (median, mL) & $2: 32(0: 42-$ & $2: 35(1: 04-$ & 0.82 \\
Operative time (median, h) & $10: 16)$ & $11: 14)$ & 0.22 \\
\hline Length of stay (median, d) & $4(2-101)$ & $4(2-35)$ & 0.22 \\
\hline
\end{tabular}

** Surgical complexity score and surgical complexity score group adapted from Aletti et al.

\section{Risk factors for pulmonary embolus}

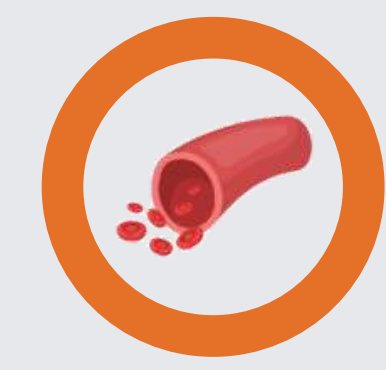

On multivariate analysis, surgery preintervention was associated with a significantly higher risk of PE.

\begin{tabular}{|lcc|}
\hline \multicolumn{3}{|c|}{ Multivariable analysis } \\
\hline $\begin{array}{l}\text { Risk factors for PEs } \\
\text { Surgery }\end{array}$ & OR (95\% Cl) & p-value \\
preintervention & $4.16(1.03-16.79)$ & 0.045 \\
EBL $\geq 500 ~ \mathbf{~ m L}$ & $2.75(1.05-7.19)$ & $\mathbf{0 . 0 4}$ \\
\hline
\end{tabular}

\begin{tabular}{|c|c|c|}
\hline \multicolumn{3}{|c|}{ Univariate analysis } \\
\hline Risk factors for PEs & OR $(95 \% \mathrm{Cl})$ & p-value \\
\hline $\begin{array}{l}\text { Surgery } \\
\text { preintervention }\end{array}$ & $4.25(1.04-17.43)$ & 0.04 \\
\hline LOS $\geq 5$ days & $11.94(2.65-53.92)$ & 0.002 \\
\hline$E B L \geq 500 \mathrm{~mL}$ & $2.85(1.05-7.78)$ & 0.04 \\
\hline $\begin{array}{l}\text { Age categories } \\
(56-70 \text { vs } \leq 55)\end{array}$ & $0.84(0.25-2.82)$ & 0.28 \\
\hline Smoking & $1.41(0.15-6.49)$ & 0.90 \\
\hline DM & $1.61(0.37-5.33)$ & 0.59 \\
\hline $\begin{array}{l}\text { Cytoreductive } \\
\text { surgery }\end{array}$ & $2.24(0.77-7.35)$ & 0.16 \\
\hline $\begin{array}{l}\text { Confirmed } \\
\text { malignancy }\end{array}$ & $0.31(0.001-1.47)$ & 0.12 \\
\hline Advanced stage & $2.22(0.69-9.38)$ & 0.23 \\
\hline $\begin{array}{l}\text { Operative time } \\
\text { ( } \geq 3 \mathrm{~h} \text { vs } 2-3 \mathrm{~h} \text { ) }\end{array}$ & $1.54(0.54-4.37)$ & 0.41 \\
\hline
\end{tabular}

\section{CONCLUSIONS}

- This dual mechanical and pharmacological thromboprophylaxis strategy resulted in a sharp decrease in risk of pulmonary embolus, from $5.1 \%$ to $0 \%$.

- Our results highlight the importance of a standardized protocol for perioperative thromboprophylaxis.

- Given the significant morbidity and mortality associated with thromboembolic events, and their highly preventable nature, guidelines and consensus specific to the Gynecologic Oncology population are warranted.

Q 2 Sunnybrook HEALTH SCIENCES CENTRE
要 Obstetrics \& Gynaecology NIVERSITY OF TORONTO 